\title{
SYNTHESIS OF NANOSILICA FROM PADDY HUSK ASH AND THEIR SURFACE FUNCTIONALIZATION
}

\author{
W.A.P.J. PREMARATNE ${ }^{1,2 *}$, W.M.G.I. PRIYADARSHANA ${ }^{2}$, \\ S.H.P. GUNAWARDENA ${ }^{3}$ AND A.A.P. DE ALWIS ${ }^{2}$
}

\section{${ }^{1}$ Department of Chemistry, University of Kelaniya, Kelaniya, Sri Lanka ${ }^{2}$ Sri Lanka Institute of Nanotechnology (SLINTEC), EPZ, Biyagama, Sri} Lanka

${ }^{3}$ Department of Chemical and Process Engineering, University of Moratuwa, Sri Lanka

\begin{abstract}
Nanosilica was synthesized by a chemical precipitation process from paddy husk ash (PHA) efficiently and effectively. Surface functionalization of the silica nanoparticles was carried out with oleic acid $\left(\mathrm{C}_{18} \mathrm{H}_{34} \mathrm{O}_{2}\right)$. Scanning electron micrographs (SEM) data showed that the nanosilica particle size was in the range of $50-70 \mathrm{~nm}$ and they were in the agglomerate form. X-ray diffraction (XRD) analysis data revealed that synthesized nanosilica was in amorphous form showing a strong broad peak at $22.14^{\circ}(2 \theta)$. Fourier transform infrared spectroscopy (FT-IR) data supported the presence of hydrogen bonded silanol group and siloxane groups in nanosilica. Surface functionalized nanosilica with oleic acid was characterized using thermogravimetric analysis (TGA) and FT-IR methods. FT-IR experimental data showed that the modified nanosilica formed the ester bonding between silanol group in nanosilica and the carboxylic group of oleic acid with a successful functionalization.
\end{abstract}

Keywords: Nanosilica; Paddy husk ash; Oleic acid; Functionalization

\footnotetext{
${ }^{*}$ Corresponding author E mail: jeewa@kln.ac.lk
} 


\section{INTRODUCTION}

Nanotechnology has attracted considerable scientific interest due to the new potential uses of particles in 1-100 nanometer scale (Stone et al. 2010). At the nanoscale, physical, chemical, and biological properties differ from the properties of individual atoms and molecules of bulk matter. Therefore, it provides opportunity to develop new classes of advanced materials which meet the demands from high-tech applications (Kharisov et al. 2010). Thus, industries may be able to re-engineer many existing products that function at unprecedented levels. A quickly growing range of applications of nanomaterials in many fields has been observed in recent years (Kharisov et al. 2010). Among them, nanosilica is a widely used nanomaterial with applications in polymer, adhesives, fiber optic strands, sealants, inks, paints, coatings, cosmetics, food additive and in cement-based building materials (Hessien et al. 2009). Nanosilica is one of the most common nanofillers used in the preparation of nanocomposites (Dorigato et al. 2012). Low valued silica bearing natural resources are converted into value added marketable types of silica by various processes (Zaky et al. 2008). The resulting silica products are generally classified based on their properties and include nanosilica, fumed silica, precipitated silica, colloidal silica and silica gels (Jal et al. 2004). The applications of nanosilica ultimately depend on the surface chemistry, molecular structure, morphology and porosities (Sun et al. 2004). Therefore, the modification of silica nanoparticles to enhance the surface chemical and physical properties is the key for the successful applications of the material (Guo et al. 2008).

Burning paddy husks as fuel to generate energy results paddy husk ash (PHA) as a waste product. PHA is rich in silica (more than 60\%) and can be used as an economically viable raw material for the production of silica and nanosilica as value added products (Kalapathy et al. 2000). At present, nanoscale silica materials are synthesized using several methods, including precipitation from sodium silicate, solgel route, templated synthesis, synthesis using emulsions, and bioinspired methods (Liou et al. 2010). It is however unclear how these methods are suited for synthesizing nanosilica for a given application by a given silica source. Furthermore, 
these synthetic methods compare with those employed industrially are required to be assessed (Kalapathy et al. 2000). As an agricultural country, Sri Lanka produces tones of paddy husk annually during the post harvesting process. The beneficiation of paddy husk has been used in many applications (Shelke et al. 2010). Therefore, the present investigation is intended to synthesize nanoslica from PHA using efficient chemical precipitation methods and characterize of nanosilica synthesized. Surface modification of nanosilica particles is carried out with oleic acid to improve the compatibility between inorganic silica nanoparticles and organic matrix for the applications in polymer engineered materials and compounds.

\section{MATERIALS AND METHODS}

White rice paddy husk ash (PHA) collected from Kaduwela area in Sri Lanka was used in this research study. Analytical grade amorphous nanosilica $(<30 \mathrm{~nm})$ from Sigma-Aldrich was used as the standard nanosilica sample. Sodium hydroxide, ammonium hydroxide, sulfuric acid, oleic acid, n-heptane and ethanol used were of analytical grade. X-ray diffraction (XRD) analysis was carried out using Brucker D8 Focus X-ray diffractometer. Scanning electron microscopy (SEM) and Energy Dispersive X-ray spectroscopy (EDX) analyses were carried by using Hitachi SU 6600 FE-SEM to ascertain morphology and the elemental composition of samples respectively. Fourier transform infrared spectroscopy (FT-IR) data were obtained from the Diffused Reflectance mode by using Bruker Vertex80 coupled with RamFT module (RAM II) spectrometer. Thermogravimetric analysis (TGA) of the samples was done using TA Instruments SDTQ600 from ambient temperature to $1200{ }^{\circ} \mathrm{C}$ at a rate of $10{ }^{\circ} \mathrm{C} / \mathrm{min}$ at nitrogen atmosphere.

\section{Synthesis of nanosilica}

Paddy husk ash (PHA) was washed by deionized water and dried at $105{ }^{\circ} \mathrm{C}$ in order to remove the moisture. Then, the dried PHA sample was burnt at $700^{\circ} \mathrm{C}$ for 6 hours. Ten grams $(10 \mathrm{~g})$ of burnt PHA was stirred in $80 \mathrm{~mL}$ of $3.0 \mathrm{M} \mathrm{NaOH}$ solution and the mixture was boiled for 3 hours. The solution was filtered and the residue was washed with $20 \mathrm{~mL}$ deionized water. The filtrate was allowed to cool down to room 


\section{W.A.P.J. Premaratne et.al.}

temperature and $2.5 \mathrm{M} \mathrm{H}_{2} \mathrm{SO}_{4}$ acid was added until the $\mathrm{pH}$ of the solution becomes pH 2 and followed by $\mathrm{NH}_{4} \mathrm{OH}$ until $\mathrm{pH} 8.5$ at room temperature. The filtrate was then dried at $120{ }^{\circ} \mathrm{C}$ for 12 hours. The resulted sample was powdered and pure silica sample was extracted by refluxing with $6 \mathrm{M} \mathrm{HCl}$ for 4 hours. Then the silica sample was washed repeatedly with deionized water until the solution become acid free. Precipitate was separated by centrifugation. The purified silica sample was dried at $105{ }^{\circ} \mathrm{C}$ for 2 hours and was subjected to EDX, XRD, FTIR, and TGA analysis.

Pure silica sample extracted from PHA was then dissolved in 3.0 $\mathrm{M} \mathrm{NaOH}$ by continuous stirring for 10 hours on a magnetic stirrer. Then $0.5 \mathrm{M} \mathrm{H}_{2} \mathrm{SO}_{4}$ was added drop-wise to adjust $\mathrm{pH}$ in the range of 7.5-8.5. The precipitated silica was washed repeatedly with warm deionized water until the filtrate became alkali free. Precipitate was separated by centrifugation (9000 rpm for 1 hour). The resulted sample was dried at $50{ }^{\circ} \mathrm{C}$ for 48 hours in order to obtain nanosilica. Finally, the nanosilica sample prepared was subjected to EDX, SEM, XRD, TGA and FTIR analyses.

The initial step is extraction of silica from Paddy husk ash (PHA) as sodium silicate using sodium hydroxide as shown in Eq. (1).

$$
\mathrm{SiO}_{2}(\text { from } \mathrm{PHA})+\mathrm{NaOH} \rightarrow \mathrm{Na}_{2} \mathrm{SiO}_{3}+\mathrm{H}_{2} \mathrm{O}
$$

In the second step of the process, silica is precipitated from sodium silicate using sulfuric acid as given in Eq. (2).

$$
\mathrm{Na}_{2} \mathrm{SiO}_{3}+\mathrm{H}_{2} \mathrm{SO}_{4} \rightarrow \mathrm{SiO}_{2}+\mathrm{Na}_{2} \mathrm{SO}_{4}+\mathrm{H}_{2} \mathrm{O}
$$

\section{Functionalization of nanosilica}

For the functionalization of nanosilica, $4 \mathrm{~mL}$ of oleic acid $\left(\mathrm{C}_{18} \mathrm{H}_{34} \mathrm{O}_{2}\right)$ was mixed with $40 \mathrm{~mL}$ of n-heptane and was stirred for 30 minutes at $1000 \mathrm{rpm}$. Then $100 \mathrm{mg}$ of silica nanoparticles was added into the solution and was stirred for another four hours at $1000 \mathrm{rpm}$ at $60{ }^{\circ} \mathrm{C}$. Oleic acid modified nanosilica particles were washed using ethanol to remove excess oleic acid and the mixture was subjected to oven dry at $60{ }^{\circ} \mathrm{C}$ until the ethyl alcohol was evaporated to obtain the final product. The functionalized nanosilica was characterized by using TGA and FTIR methods. 


\section{RESULTS AND DISCUSSION}

Energy dispersive X-ray spectroscopy (EDX) analysis

Table 1: EDX analysis of different products at different steps in nanosilica synthesis process

\begin{tabular}{|c|c|c|c|c|c|}
\hline \multirow{2}{*}{ Element } & \multicolumn{5}{|c|}{ Weight percentage (\%) } \\
\cline { 2 - 6 } & $\begin{array}{c}\text { PHA after } \\
\text { burnt at } \\
\mathbf{7 0 0}{ }^{\circ} \mathrm{C}\end{array}$ & $\begin{array}{c}\text { Before reflux } \\
\text { with HCl }\end{array}$ & $\begin{array}{c}\text { After reflux } \\
\text { with HCl }\end{array}$ & Nanosilica & $\begin{array}{c}\text { Oleic acid } \\
\text { functionalized } \\
\text { nanosilica }\end{array}$ \\
\hline $\mathrm{C}$ & 4.64 & 3.55 & 3.40 & 3.41 & 10.53 \\
\hline $\mathrm{O}$ & 55.01 & 43.81 & 56.02 & 55.52 & 50.82 \\
\hline $\mathrm{Na}$ & - & 25.02 & - & - & - \\
\hline $\mathrm{Mg}$ & 0.59 & - & - & - & - \\
\hline $\mathrm{Al}$ & 0.77 & 0.71 & - & & 38.65 \\
\hline $\mathrm{Si}$ & 35.47 & 13.05 & 40.58 & 41.07 & - \\
\hline $\mathrm{P}$ & 0.94 & - & - & - & - \\
\hline $\mathrm{Cl}$ & 0.09 & - & - & - & - \\
\hline $\mathrm{K}$ & 1.73 & 0.51 & - & - & - \\
\hline $\mathrm{Ca}$ & 0.53 & - & - & - & - \\
\hline $\mathrm{Fe}$ & 0.21 & - & - & - & - \\
\hline $\mathrm{S}$ & - & 13.35 & & - & - \\
\hline
\end{tabular}

Table 1 presents the chemical composition of different products at different steps throughout the synthesis process of nanosilica from PHA. According to EDX chemical analysis data, synthesized nanosilica has high chemical purity. However, $3.4 \%$ carbon can be detected in synthesized nanosilica as impurities which had come during the sample preparation step. In addition, oleic acid functionalized nanosilica has given higher percentage of carbon (10.5\%) compared to the nanosilica during 


\section{W.A.P.J. Premaratne et.al.}

$\mathrm{EDX}$ analysis. The intermediate silica product, before $\mathrm{HCl}$ acid treatment, contained $25 \%$ of $\mathrm{Na}$ and $13.4 \%$ of $\mathrm{S}$ forming $\mathrm{Na}_{2} \mathrm{SO}_{4}$ compound (Indumathi et al. 2011). However, the intermediate silica product after $\mathrm{HCl}$ acid treatment followed by a washing cycle gave only $40.6 \%$ of silican, $56 \%$ of oxygen and $3.4 \%$ of carbon. The percentage yield of nanosilica synthesized from burnt PHA at $700{ }^{\circ} \mathrm{C}$ was $64 \%$.

\section{XRD analysis}
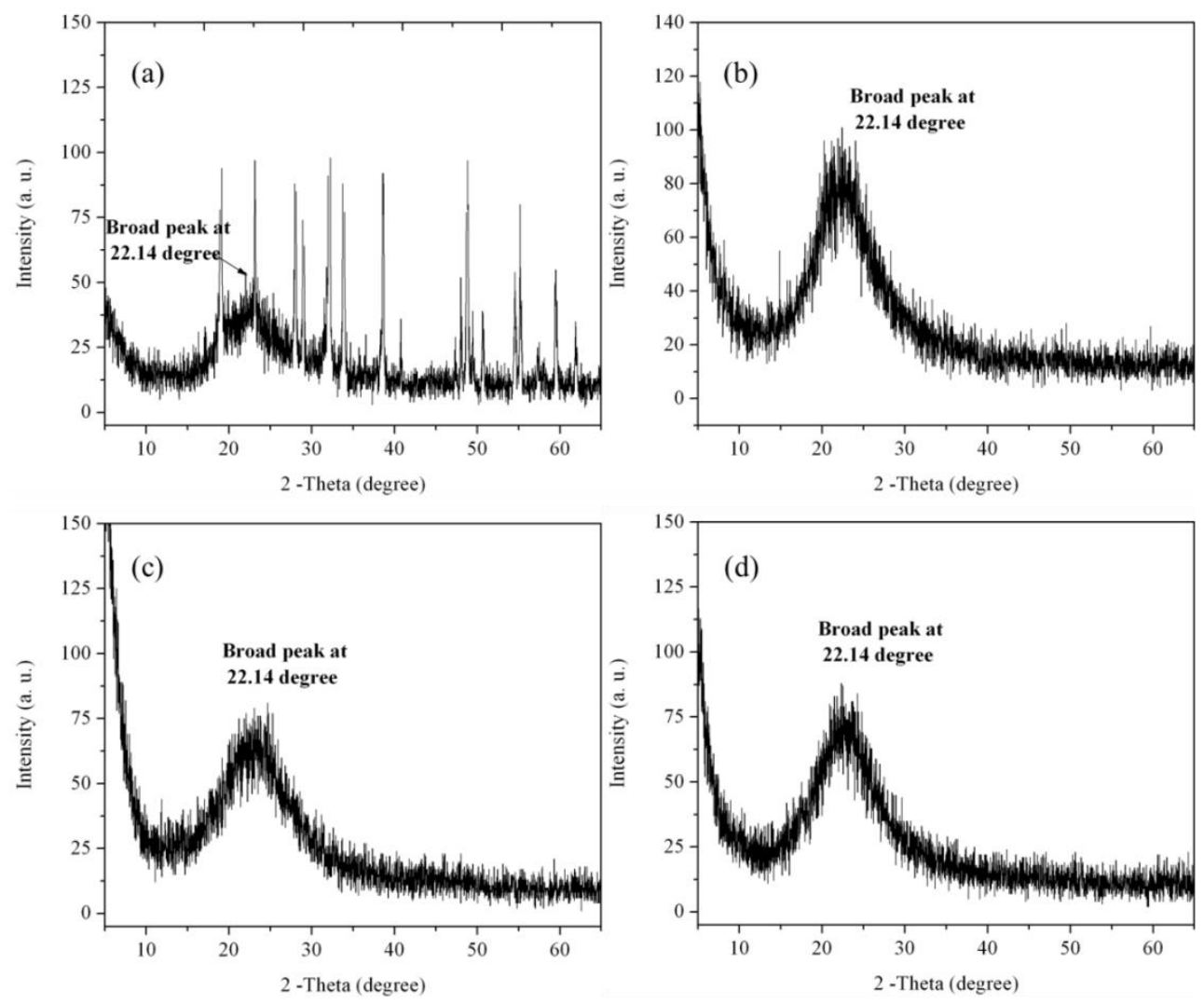

Figure 1: XRD patterns of (a) silica prior to $\mathrm{HCl}$ acid treatment, (b) silica after $\mathrm{HCl}$ acid treatment, (c) nanosilica from PHA and, (d) standard nanosilica

Figure 1 shows the powder X-ray diffraction patterns of silica in the intermediate stage before refluxing with $\mathrm{HCl}$ (a), silica in the intermediate stage after 
refluxing with $\mathrm{HCl}$ (b), nanosilica synthesized from PHA (c), and standard amorphous nanosilica $(<30 \mathrm{~nm})$ from Sigma-Aldrich (d). Figure 2(a) shows a different XRD diffractogram due to the crystalline structural peaks of sodium sulfate $\left(\mathrm{Na}_{2} \mathrm{SO}_{4}\right)$, which has formed as a result of the reaction between sodium silicate and sulfuric acid. It can be removed by a simple washing process. XRD diffractograms of nanosilica synthesized from PHA in Figure 1(c) and standard nanosilica in Figure 1(d) showed strong board peaks at $22.14^{\circ}(2 \theta)$ which is characteristic of amorphous silica (Singh et al. 2008). Therefore, nanolisica synthesized from PHA is purely amorphous type.

\section{SEM analysis}
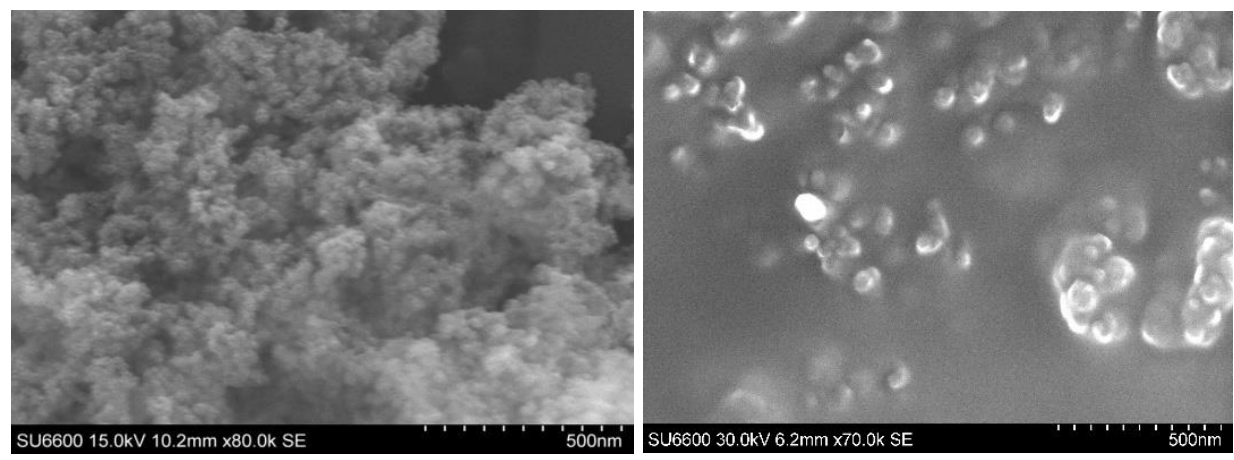

Figure 2: SEM images of the nanosilica synthesized from PHA

SEM images given in Figure 2 show the morphology and the particle size of the silica nanoparticles synthesized form PHA. According to the SEM images obtained at $15 \mathrm{kV}$ and $30 \mathrm{kV}$, the particle size of the nanosilica was found to be in the range of 50-70 $\mathrm{nm}$. The nanosilica particles do not show clear boundaries as they are in agglomerate and amorphous form. Therefore, SEM data confirmed that the size of the silica particles synthesized from PHA is in the nanorange (1-100 nm). 


\section{Thermogravimetric analysis}

The thermogravimetric analysis (TGA) and the derivatives curves of different products at different stages in nanosilica synthesis process and the standard nanosilica $(<30 \mathrm{~nm})$ are shown in Figure 3. In Figure 3 (a), approximately $40 \%$ weight loss can be observed in the intermediate silica product prior to $\mathrm{HCl}$ acid treatment due to $\mathrm{Na}_{2} \mathrm{SO}_{4}$ formed during the reaction. However, the thermogram given in Figure 3(b) does not show a significant weight between $1100{ }^{\circ} \mathrm{C}-1200{ }^{\circ} \mathrm{C}$ compared to Figure3(a), as the excess $\mathrm{Na}_{2} \mathrm{SO}_{4}$ has been removed with the acid treatment followed by a washing cycles. There are two distinct mass loss steps in thomograms in Figure 3(c) and Figure 3(d). The first step (less than $130{ }^{\circ} \mathrm{C}$ ) is abrupt and is most probably due to the removal of physisorbed water from the silica surface (Ek et al. 2001). The second step is border and is considered to correspond to slow condensation of silanols (Ek et al. 2001). Therefore, the TGA data showed that the synthesized nanosilica from PHA has the same characteristic properties compared to the standard amorphous nanosilica sample ( $<30 \mathrm{~nm}$ ) from Sigma-Aldrich.

TGA and the derivative curves of nanosilica synthesized from PHA, pure oleic acid and, oleic acid functionalized nanosilica are shown in Figure 4. In Figure 4(a), the percentage weight loss of nanosilica synthesized from PHA is $16 \%$ whereas the percentage weight loss of oleic acid functionalized nanosilica is 9\% in Figure 4(c) at $117{ }^{\circ} \mathrm{C}$ temperature. Those weight losses are due to the removal of moisture adsorbed on the surface hydroxyl groups of nanosilica. Therefore, TGA results showed that oleic acid functionalized nanosilica made less water absorption than the pure nanosilica sample indicating $7 \%$ weight loss difference between pure nanosilica and oleic acid functionalized nanosilica at $117^{\circ} \mathrm{C}$ temperature. According to Figure 4(b) oleic acid is decomposed before $500{ }^{\circ} \mathrm{C}$ temperature. When nanosilica particles are functionalized with oleic acid, surface hydroxyl groups form ester bonds through carboxylic groups of oleic acid resulting hydrophobic surface which do not facilitate water molecules to be adsorbed on to the surface of the nanosilica. Still there are number of hydroxyl groups which are not modified by oleic acid. Therefore, water molecules can attach to those areas where free hydroxyl groups are existed. 

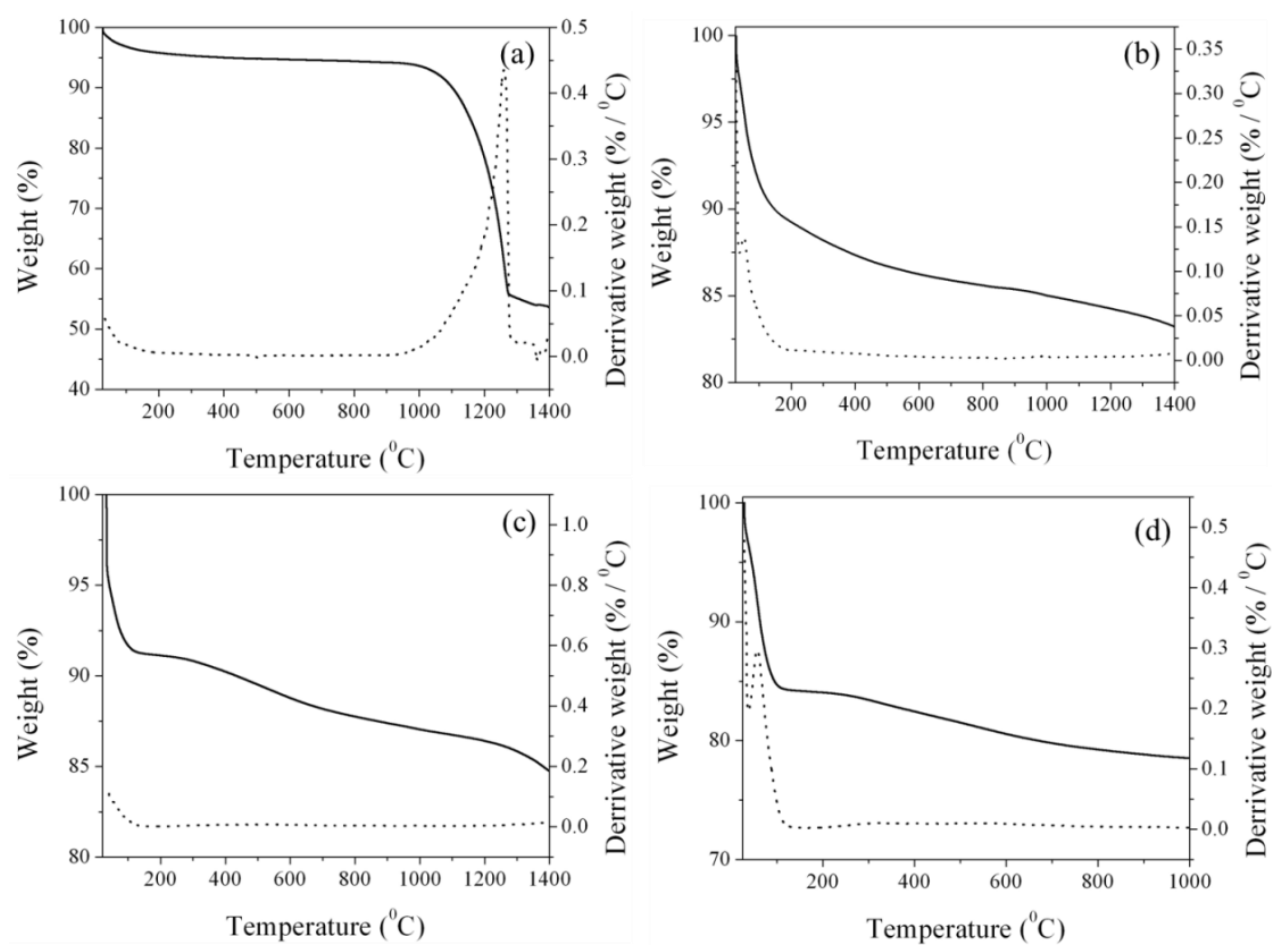

Figure 3: TGA and the derivative curves of (a) silica prior to $\mathrm{HCl}$ acid treatment, (b) silica after $\mathrm{HCl}$ acid treatment, (c) nanosilica from PHA and, (d) standard nanosilica

According to the thermogram given in Figure 4(c), there is a significant weight loss between $400{ }^{\circ} \mathrm{C}$ and $500{ }^{\circ} \mathrm{C}$ in oleic acid functionalized nanosilica compared to unmodified nanosilica in Figure 4(a). This is mainly due to the decomposition of oleic acid from the surface of the functionalized nanosilica. TGA data showed that the functionalization of nanosilica with oleic acid has been occurred successfully. 
W.A.P.J. Premaratne et.al.
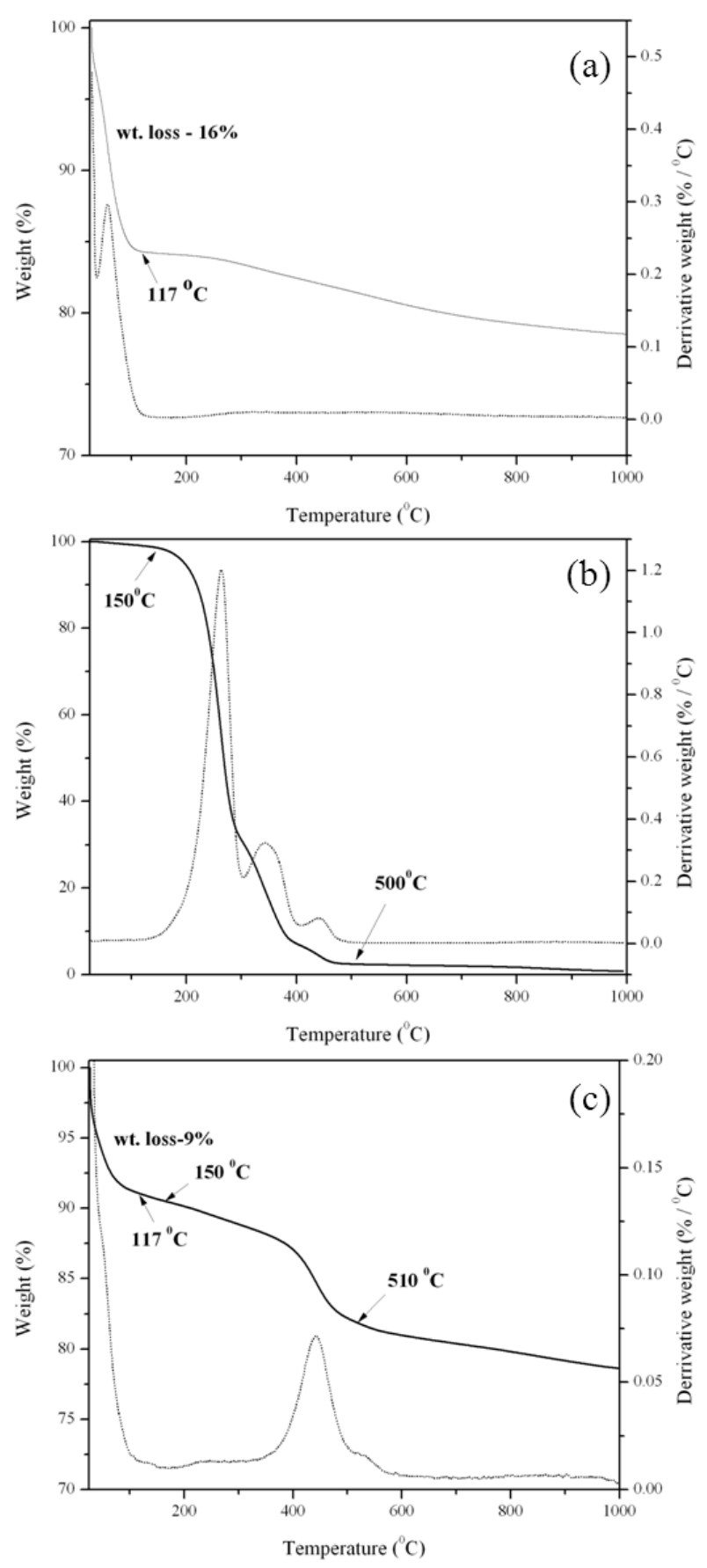

Figure 4: TGA and the derivative cures of (a) nanosilica from PHA, (b) pure oleic acid and, (c) oleic acid functionalized nanosilica 


\section{FT-IR spectroscopic analysis}

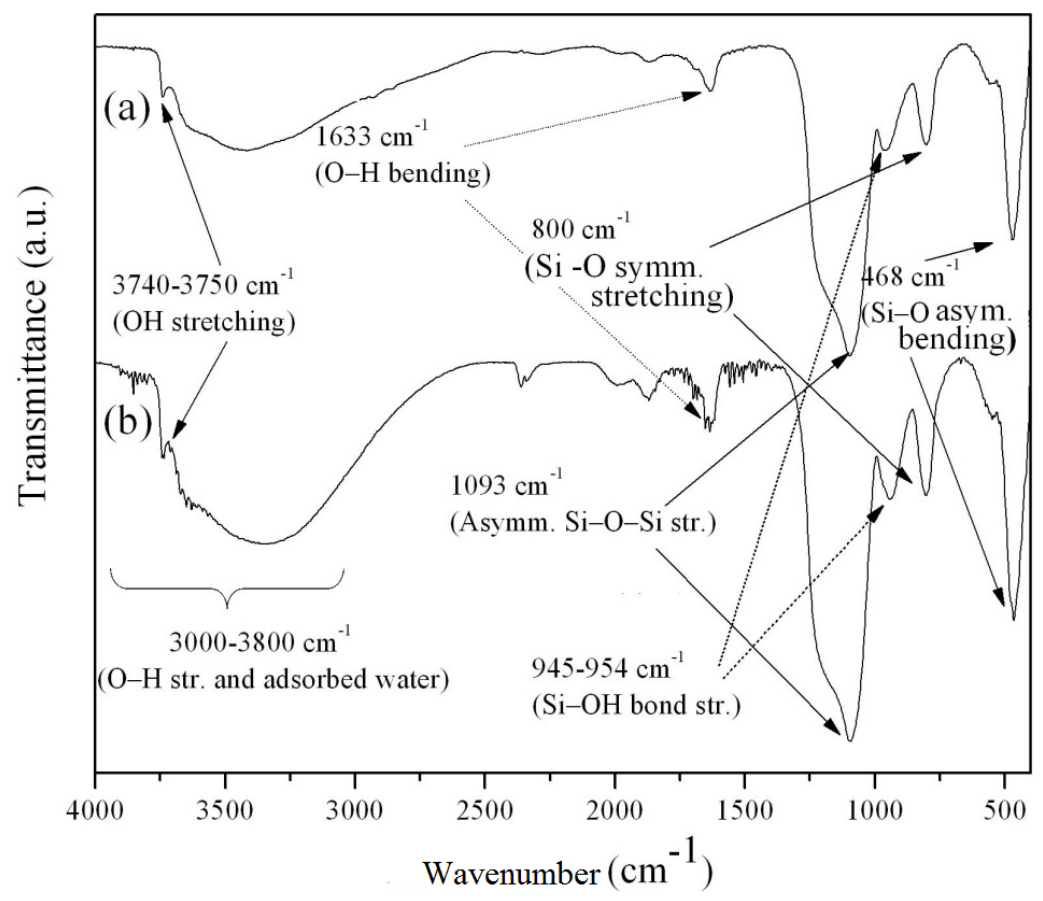

Figure 5: FT-IR spectra of (a) nanosilica from PHA and, (b) standard nanosilica

Figure 5 shows the FT-IR spectra of nanosilica synthesized from PHA (a) and standard amorphous nanosilica sample $(<30 \mathrm{~nm})$ from Sigma-Aldrich (b). The broad band in the range of $3800-3000 \mathrm{~cm}^{-1}$ region is mainly due to $\mathrm{O}-\mathrm{H}$ stretching vibration of different kinds of hydroxyl (-OH) groups such as silanol hydroxyl groups. Research studies by Zhou et al. (2001) also indicated that -OH peak broadening can happen due to physically and chemically adsorbed water molecules on the $\mathrm{SiO}_{2}$ surface. The moderately sharp peak at $3750-3740 \mathrm{~cm}^{-1}$ region is assigned to the stretching frequency of hydroxyl group and the peak at $1633 \mathrm{~cm}^{-1}$ is attributed to the bending vibration of the $\mathrm{O}-\mathrm{H}$ bonds in adsorbed water molecule on the surface of nanosilica. The peak at $1093 \mathrm{~cm}^{-1}$ is assigned to $\mathrm{Si}-\mathrm{O}-\mathrm{Si}$ asymmetric stretching vibration and the band at $800 \mathrm{~cm}^{-1}$ is due to the symmetric stretching vibration of the $\mathrm{Si}-\mathrm{O}-\mathrm{Si}$ bond. The peak appeared at $468 \mathrm{~cm}^{-1}$ is related to bending vibration peak of the Si-O-Si bond (Guo et al. 2008). Therefore, FT-IR spectrum of nanosilica 


\section{W.A.P.J. Premaratne et.al.}

synthesized from PHA clearly indicated that they are in amorphous form compared with the standard amorphous nanosilica sample given in Figure 5(b).
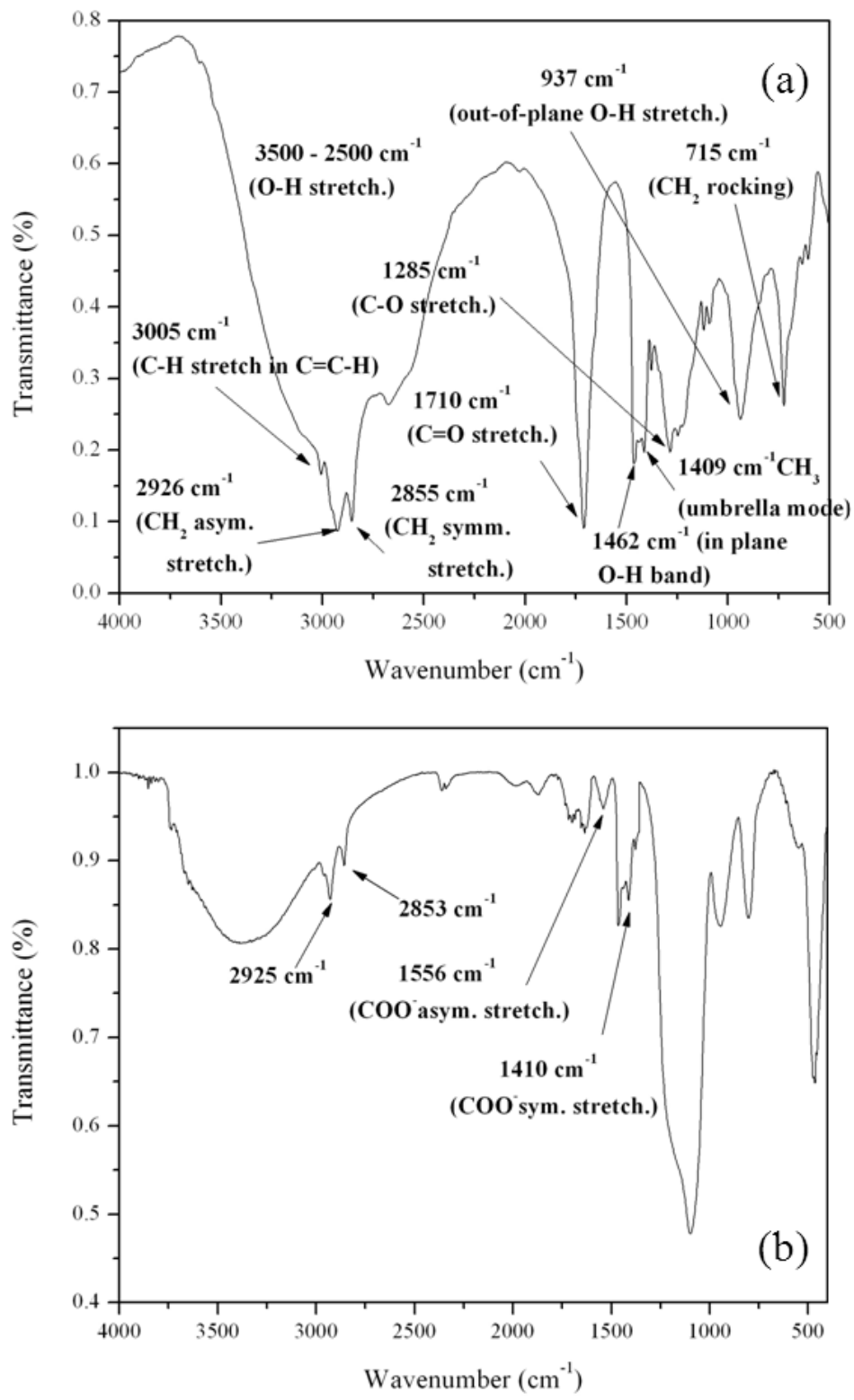

Figure 6: FTIR spectra of (a) oleic acid and, (b) oleic acid functionalized nanosilica

In order to get a better understanding of the surface functionalization of the oleic acid on the surface of silica nanoparticles, FT-IR measurements were carried 


\section{Synthesis of nanosilica from paddy husk ash}

out on pure oleic acid and the nanosilica fictionalized with oleic acid. Figure 6(a) shows the FT-IR spectrum of oleic acid. The broad peak in the $3500-2500 \mathrm{~cm}^{-1}$ region is due to the O-H stretching of carboxylic acid groups (-COOH) (Kooter et al. 1997). Two sharp peaks appeared at $2855 \mathrm{~cm}^{-1}$ and $2926 \mathrm{~cm}^{-1}$ represent symmetric and asymmetric $-\mathrm{CH}_{2}$ stretching vibrations respectively (Hong et al., 2009). The moderately sharp peak at $3005 \mathrm{~cm}^{-1}$, which is superimposed with $\mathrm{O}-\mathrm{H}$ stretching band, is attributed to the C-H stretching in vinyl moiety (Kooter et al. 1997). The sharp peak appeared at $1710 \mathrm{~cm}^{-1}$ is mainly due to the $\mathrm{C}=\mathrm{O}$ stretching and the peak appeared at $1285 \mathrm{~cm}^{-1}$ is attributed to the C-O stretching of carboxylic group (Kooter et al. 1997).

In Figure 6(b) and Figure 5(a), both modified and unmodified nanosilica showed the bands at 1093, 800 and $468 \mathrm{~cm}^{-1}$ which can be assigned to -Si-O-Si- vibration modes (Guo et al. 2008). In Figure 6(b), two new peaks appearing at 1410 and $1556 \mathrm{~cm}^{-1}$ are attributed to the symmetric and asymmetric stretching vibrations of COO- (Hong et al. 2009), while no such peaks can found in nanosilica in Figure 5(a). Oleic acid functionalized nanosilica indicated a smaller peak at $1717 \mathrm{~cm}^{-1}$, which confirmed the ester bonding between silinol group and the carboxylic group of the oleic acid. Therefore, it can be concluded that the $-\mathrm{COOH}$ of the oleic acid has reacted with the surface $-\mathrm{OH}$ groups of nanosilica to form carboxylate bond (Pishvaei et al. 2010). In addition, the absorption peaks at 2853 and $2925 \mathrm{~cm}^{-1}$ in Figure 6(b) are characteristic of stretching vibrations of $-\mathrm{CH}_{2}$ group (Hong et al. 2009), which are not observed in pure nanosilica in Figure 5(a). It shows that long alkyl chains are present in the modified nanosilica with oleic acid. Therefore, FT-IR data revealed that silica nanoparticles have been successfully functionalized by oleic acid.

\section{CONCLUSIONS}

Nanosilica was synthesized from paddy husk ash (PHA) efficiently and effectively and was characterized by various analytical techniques. The percentage yield of nanosilica synthesized from burnt PHA at $700{ }^{\circ} \mathrm{C}$ was $64 \%$. SEM analysis data showed that the nanosilica particles from PHA were in the agglomerate form and the particle size was in the range of 50-70 $\mathrm{nm}$. The particle shape was found to be 
uniform. X-ray diffractograms showed a strong broad peak at $22.14^{\circ}(2 \theta)$ indicating nanosilica synthesized from PHA was amorphous. FTIR data revealed the presence of hydrogen bonded silanol group and siloxane groups in silica. Silica nanoparticles were subjected to surface functionalization with oleic acid and characterized using TGA and FT-IR methods. TGA data indicated the thermal decomposition of oleic acid from the surface of functionalized nanosilica with a significant weight loss between $400{ }^{\circ} \mathrm{C}$ to $500{ }^{\circ} \mathrm{C}$. FTIR experimental data showed that the modified nanosilica has the ester bonding between silanol group and the carboxylic group of the oleic acid. Therefore, TGA and FT-IR data revealed that silica nanoparticles have been successfully functionalized by oleic acid.

\section{REFERENCES}

Dorigato A., Sebastiani M. and Pegoretti A., 2012. Effect of silica nanoparticle on the mechanical performances of poly (lactic acid). Journal of Polymers and Environment 20: 713-725.

Ek S., Root A., Peussa M. and L. Niinisto., 2001. Determination of the hydroxyl group content in silica by thermogravimetry and a comparison with ${ }^{1} \mathrm{H}$ MAS NMR results. Thermochimica Acta 379: 201-212.

Guo Y., Wang M., Zhang H., Liu G., Zhang L. and Qu X., 2008. The surface modification of nanosilica, preparation of nanosilica/acrylic core-shell latex, and is application in toughening PVC matrix. Journal of Applied Polymer Science 107: 2671-2680.

Hessien M. M., Rashad M. M., Zaky R. R., Abdel-Aal E. A. and El-Barawy K. A., 2009. Controlling the synthesis conditions for silica nanosphere from semiburned rice straw. Materials Science and Engineering B 162: 14-21.

Hong R.Y., Li J.H., Zhang S.Z., Li H. Z., Zheng Y., Ding J. and Wei D.J., 2009. Preparation and characterization of silica coated $\mathrm{Fe}_{3} \mathrm{O}_{4}$ nanoparticles used as precursor of ferrofluids. Applied Surface Science 255: 3485-3492.

Indumathi P., Shabhudeen S.P. and Saraswathy C.P., 2011. Synthesis and characterization of nano silica from the Pods of Delonix Regia ash. International Journal of Advanced Engineering Technology 2(4): 421-426. 
Jal P.K., Sudarshan M., Saha A., Patel S. and Mishra B.K., 2004. Synthesis and characterization of nanosilica prepared by precipitation method. Colloids and Surfaces A: Physicochemical and Engineering Aspects 240: 173-178.

Kalapathy U., Proctor A. and Shultz J., 2000. A simple method for production of pure silica from rice hull ash. Bioresource Technology 73: 257-262.

Kharisov B.I., Kharissova O.V. and Jose-Yacaman M., 2010. Nanostructures with animal like shapes. Industrial and Engineering Chemistry Research 49: 8289-8309.

Kooter I.M., Pierik A.J., Merkx M., Averill B.A., Moguilevsky N., Bollen A. and Wever R., 1997. Difference Fourier Transform Infrared evidence for ester bonds linking the Heme group in myeloperoxidase, lactoperoxidase, and eosinophil peroxidase. Journal of American Chemical Society 119: 1154211543.

Liou T.H. and Wu S.J., 2010. Kinetics study and characteristics of silica nanoparticles produced from biomass-based material. Industrial and Engineering Chemistry Research 49: 8379-8387.

Pishvaei M. and Tabrizi F.F., 2010. Synthesis of high solid content polyacrylate/nanosilica latex via miniemulsion polymerization. Iranian Polymer Journal 19(9): 707-716.

Shelke V.R., Bhagade S.S. and Mandavgane S.A., 2010. Mesoporous silica from rice husk ash. Bulletin of Chemical Engineering \& Catalysis 5(2): 63-67.

Singh D., Kumar R., Kumar A. and Rai K. N., 2008. Synthesis and characterization of rice husk silica, silica-carbon composite and $\mathrm{H}_{3} \mathrm{PO}_{4}$ activated silica. Ceramica 54: 203-212.

Stone V., Nowack B., Baun A., Brink N.V.D., Kammer F.V.D, Dusinska M., Handy R., Hankin S., Hassellov M., Joner E. and Fernandes T.F., 2010. Nanomaterials for environmental studies: Classification, reference material issues, and strategies for physico-chemical characterization. Science of the Total Environment 408: 1745-1754.

Sun Q., Vrieling E.G., Van-Santen R.A. and Sommerdijk N.A.J.M., 2004. Current Opinion in Solid State and Material Science 8: 111-120. 
Zaky R. R., Hessien M. M., El-Midany A. A., Khedr M. H., Abdel-Aal E. A. and ElBarawy K. A., 2008. Preparation of silica nanoparticles from semi-burned rice straw ash. Powder Technology 185: 31-35.

Zhou Z.F., Huang H.and Liu N.C., 2001. Kinetics and mechanism of grafting of oleic acid onto crylonitrile-butadiene styrene polymer. European Polymer Journal 37: 1967-1974. 